\title{
Editorial
}

\section{Nada es blanco o negro.}

En medicina nada es blanco o negro, teniendo presente el componente biológico de las enfermedades, se requiere analizar las diferentes etapas y comportamientos de éstas.

Ya a comienzos del siglo 20 Sir Hamilton Bailey describe la presencia de una nueva entidad donde la presencia de una metástasis umbilical tendría un origen neoplásico abdominal, con una incidencia de 1$3 \%$, a la cual denominó "Nódulo de la Hermana Maria Joseph" en inglés: "Sister Mary Joseph's nodule"1; De esta manera las neoplasias malignas presentan diferentes conductas que debemos tener presente al momento del diagnóstico o recurrencia que nos permitirá escoger el tratamiento y seguimiento más adecuado.

En el trabajo de Rueda et al, Metástasis gástrica de carcinoma endometrial, una localización inusual, se presenta una metástasis de localización infrecuente, 7 años posterior a su tratamiento, la cual es una recurrencia poco frecuente, y en un tardío periodo de tiempo, debido a que el $80 \%$ de las recurrencias se presentan dentro de los primeros 2 años ${ }^{2}$.

Al analizar las localizaciones inusuales de los tumores ginecológicos podemos mencionar que en el cáncer de cérvix se presentan metástasis pulmonares $5 \%$, linfonodos para áorticos aislados 1,7\% y metástasis cutáneas 0,1 a $2 \%{ }^{3}$ (más frecuente en la histología de adenocarcinoma). En cáncer de ovario se pueden observar metástasis óseas 3,7\%, cerebrales 0,3 a $2,2 \%$, mama 0,03 - 0,6\% o linfáticas extra abdominales 2 - $4 \%{ }^{4}$.

Las metástasis inusuales del cáncer de endometrio se presentan como lesiones cutaneas $0,8 \%$, linfonodos extra abdominales $0,4-1 \%$, cerebrales $1 \%$, oseas 4 $\%$ y músculo esquelético $2-4 \% 5$.
Al analizar este caso clínico con una recurrencia posterior a su tratamiento primario, se nos presentan grupos de pacientes que tendrán pronósticos y tratamientos diferentes. Hay un grupo que podemos considerar virgenes a quimioterapia, en el cual el esquema de preferencia es Carboplatino y Paclitaxel (GOG 209) por sobre el de triple droga con Doxorrubicina (TAP); ya que, si bien ambos esquemas de tratamiento presentan una progresión libre de enfermedad y sobrevida global similar, el de bidroga presenta una menor toxicidad grado $2^{6}$.

El otro grupo son las pacientes que han presentado progresión posterior a la quimioterapia a las cuales se les puede tratar con inmunoterpaia. En éste se debe evaluar la presencia de MMR (mismatch repair) en la biopsia primaria y en la de la nueva metástasis. En las mujeres que presentan un deficit de MMR o inestabilidad microsatelite se puede indicar pembrolizumab7. Esto último basado en la clasificación según el TCGA (The Cancer Genome Atlas o El Atlas del genoma del cáncer) en: 1) Tumores POLE (ultramutados) 2) Tumores de inestabilidad microsatelite, 3) Tumores con bajo número de copias con mutaciones de TP53 y 4) Tumores con alto número de copias con mutaciones de TP538.

Para pacientes con MMR intacto se sugiere el uso de una segunda linea de quimioterapia que dependerá del intervalo libre de enfermedad. Cuando es mayor a 6 meses, se sugiere la implementación de quimioterapia en base a platino. Cuando es menor a 6 meses se sugiere el uso combinado o monodroaga de pembrolizumab/lenvatinib (Inhibidor del receptor VEGF). En mujeres que no puedan recibir quimioterapia por una patología asociada o riesgo de toxicidad se puede indicar terapia endocrina (progestina) con una remisión parcial de un 15 - 30\% y 
una progresión libre de enfermedad de hasta 2 años 5,9 .

Desde la implementación de guías clínicas y protocolos, el estudio de las pacientes se ha estandarizado, lo que permite mejorar la calidad de atención y tratamientos, pero ha dejado de lado un pensamiento para argumentar e innovador, olvidando el comportamiento biológico de las enfermedades, lo que nos puede llevar a administrar erróneamente $u$ omitir tratamientos.

Este trabajo nos refuerza y recuerda que debemos tomar una pausa y analizar en detalle la historia clínica para beneficiar la calidad de vida y tratamiento de las pacientes oncológicas.

\section{REFERENCIAS}

1. Giner Galvañ V. [Sister Mary Joseph's nodule. Its clinical significance and management]. An Med Interna. 1999 Jul;16(7):365-70.

2. Zola P, Macchi C, Cibula D, Colombo N, Kimmig $\mathrm{R}$, Maggino $\mathrm{T}$, et al. Follow-up in Gynecological Malignancies: A State of Art. Int J Gynecol Cancer. 2015 Sep;25(7):1151-64.

3. Unusual Metastasis from Carcinoma Cervix [Internet]. [cited 2020 Mar 13]. Available from: https://www.ncbi.nlm.nih.gov/pmc/articles/PMC49 58070/

4. Thomakos N, Diakosavvas $M$, Machairiotis $N$, Fasoulakis Z, Zarogoulidis P, Rodolakis A. Rare Distant Metastatic Disease of Ovarian and Peritoneal Carcinomatosis: A Review of the Literature. Cancers (Basel) [Internet]. 2019 Jul 24 [cited 2020 Mar 13];11(8). Available from: https://www.ncbi.nlm.nih.gov/pmc/articles/PMC67 21345/

5. Colombo N, Creutzberg C, Amant F, Bosse T, González-Martín A, Ledermann J, et al. ESMOESGO-ESTRO Consensus Conference on Endometrial Cancer: diagnosis, treatment and follow-up. Ann Oncol. 2016 Jan;27(1):16-41.

6. Late-Breaking Abstract 1: Randomized phase III noninferiority trial of first line chemotherapy for metastatic or recurrent endometrial carcinoma: A Gynecologic Oncology Group study - Gynecologic Oncology [Internet]. [cited 2020 Mar 13]. Available from: https://www.gynecologiconcologyonline.net/article/S0090-8258(12)00228-

4/abstract
7. Howitt BE, Shukla SA, Sholl LM, Ritterhouse LL, Watkins JC, Rodig S, et al. Association of Polymerase e-Mutated and Microsatellite-Instable Endometrial Cancers With Neoantigen Load, Number of Tumor-Infiltrating Lymphocytes, and Expression of PD-1 and PD-L1. JAMA Oncol. 2015 Dec;1(9):1319-23.

8. Talhouk A, McAlpine JN. New classification of endometrial cancers: the development and potential applications of genomic-based classification in research and clinical care. Gynecol Oncol Res Pract. 2016;3:14.

9. Markman M. Hormonal therapy of endometrial cancer. Eur J Cancer. 2005 Mar;41(5):673-5.

\section{Dr. Roberto Altamirano Assad \\ Ginecología Oncológica.}

Prof. Asistente(O).

Departamento de Obstetricia y Ginecología. Facultad de Medicina Universidad de Chile. Miembro Directorio SOCHOG 